\title{
A Hybrid Combination Scheme for Cooperative Spectrum Sensing in Cognitive Radio Networks
}

\author{
Changhua Yao and Qihui Wu \\ Institute of Communications Engineering, PLA University of Science and Technology, Nanjing, China \\ Correspondence should be addressed to Changhua Yao; ych2347@163.com
}

Received 23 October 2013; Revised 20 January 2014; Accepted 22 January 2014; Published 6 March 2014

Academic Editor: Linying Xiang

Copyright (C) 2014 C. Yao and Q. Wu. This is an open access article distributed under the Creative Commons Attribution License, which permits unrestricted use, distribution, and reproduction in any medium, provided the original work is properly cited.

\begin{abstract}
We propose a novel hybrid combination scheme in cooperative spectrum sensing (CSS), which utilizes the diversity of reporting channels to achieve better throughput performance. Secondary users (SUs) with good reporting channel quality transmit quantized local observation statistics to fusion center (FC), while others report their local decisions. FC makes the final decision by carrying out hybrid combination. We derive the closed-form expressions of throughput and detection performance as a function of the number of SUs which report local observation statistics. The simulation and numerical results show that the hybrid combination scheme can achieve better throughput performance than hard combination scheme and soft combination scheme.
\end{abstract}

\section{Introduction}

Cognitive radio (CR) is a promising technology to combat spectrum scarcity by exploiting the unused spectrum bands licensed to the primary user (PU) [1,2]. There are many researches about spectrum sensing which plays a key role in cognitive radio systems and collaborative spectrum sensing schemes that have been proposed to improve the reliability of sensing [3-8], but it also introduces additional information exchange which leads to reporting overhead. In the centralized cooperative energy sensing, the hard combination scheme collects 1-bit local decision, while the soft combination scheme collects multibit information of the detected energy, which provides a better performance than the hard combination scheme at the cost of increased reporting overhead. The reporting overhead cannot be ignored when the local sensing data is reported to the FC through a common control channel (reporting channel), which is stringent bandwidth-limited for the efficient usage of limited spectrum resource $[9,10]$.

There are some researches which investigate overheadthroughput tradeoff in cognitive radio network (CRN) that consists of a number of the SUs employing energy detectors and a single decision FC. Many methods have been proposed to reduce the reporting overhead. Some introduce multibit quantization combining scheme [11], to reduce the amount of transmitted data, and some focus on the selection of the collaborative users [12]. To our best knowledge, all the related researches adopt either hard combination scheme or soft combination scheme and assume that the data rates on the reporting channels between SU and FC are all the same. In fact, the attenuations of reporting channels are hardly the same [9]. To meet the bit error rate (BER) required by the reliable communication, the data rates on the reporting channels would be different. In hard combination scheme, SUs with high reporting data rate waste their reporting capability, and in soft combination scheme, SUs with low reporting data rate introduce large overhead.

In this letter, we propose a novel hybrid combination scheme to achieve better throughput performance. SU transmits either local observation statistics or local decision to FC. FC makes the final decision by carrying out hybrid combination. The theoretical analysis and simulation show that the hybrid combination scheme outperforms hard combination and soft combination scheme.

\section{System Model}

We consider the scenario illustrated in Figure $1 . \mathrm{SU}_{i}(i=$ $1,2, \ldots, M$ where $M$ denotes the number of SUs) is the independent energy detector and reports its local decision or its quantized local observation statistics to $\mathrm{FC}$ via common 


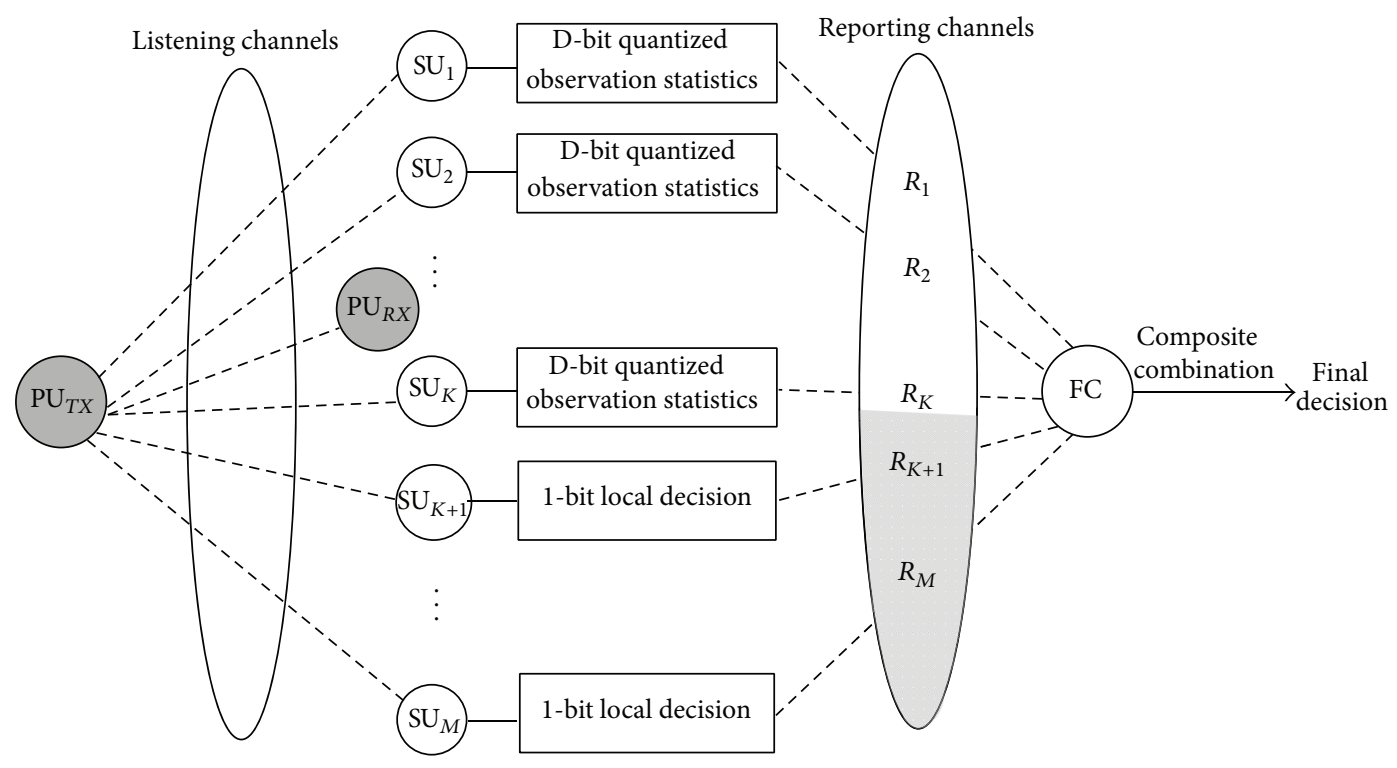

FIGURE 1: System model of hybrid combination scheme.

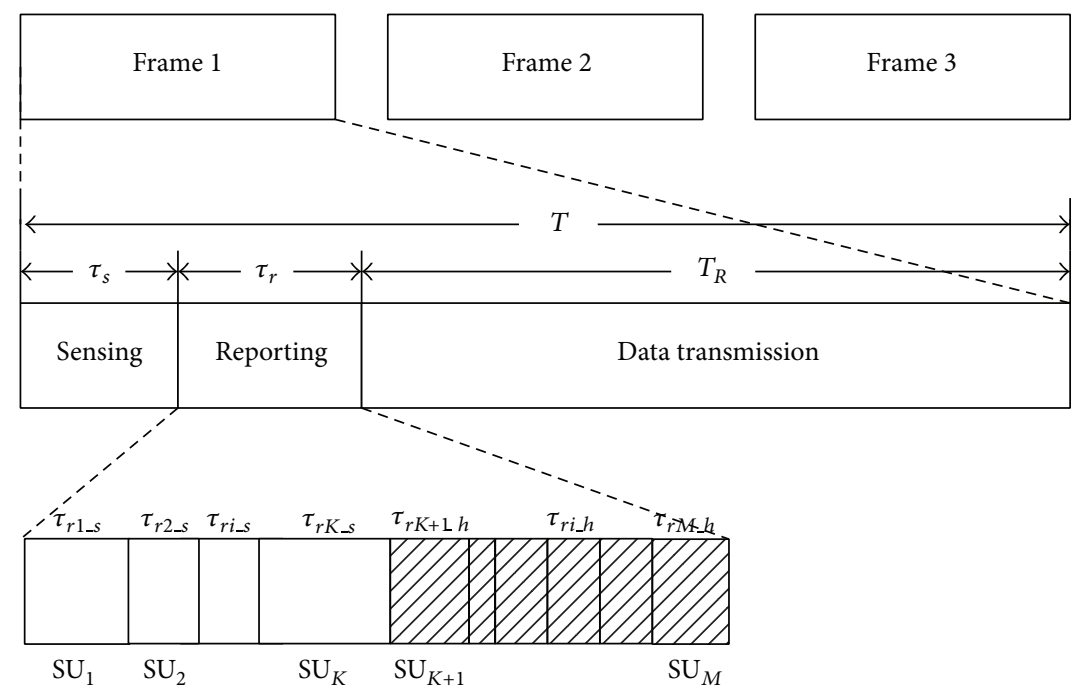

FIGURE 2: Frame structure of hybrid combination scheme.

control channel (reporting channel) sequentially [9]. Though the bandwidth of reporting channel is determined by the resource allocation, the quality of reporting channels is different. For example, in Figure $1, \mathrm{SU}_{i}(i=K+1, K+$ $2, \ldots, M)$ are shadowed over the reporting channel. When the cooperative relationship between SUs and FC is set up, they should negotiate the data rate on the reporting channel according to the quality of reporting channel. We do not focus on the relationship among signal-to-noise ratio (SNR), modulation, BER, and data rate on the reporting link; just simply assume that the data rate is $R_{i}$ when $\mathrm{SU}_{i}$ transmits data on the reporting channel under the BER constraint to make the reporting reliable. We denote vector $R=\left[R_{1}, R_{2}, \ldots, R_{M}\right]$ as the descending order of reporting data rates. After the negotiation, reporting rate vector $R$ can be obtained by the FC. In the hybrid combination scenario proposed, $\mathrm{SU}_{i}$ ( $i=K+1, K+2, \ldots, M)$ with bad reporting channel reports local decision to $\mathrm{FC} ; \mathrm{SU}_{i}(i=1,2, \ldots, K)$ with good reporting channel reports local observation statistics to FC. FC makes the final decision by carrying out hybrid combination.

The frame structure for the periodic spectrum sensing is shown in Figure 2. All the SUs perform local spectrum sensing simultaneously in the sensing phase and report local data to the FC sequentially in the reporting phase. We assume that the duration of the sensing phase is $\tau_{s}$, the duration of the reporting phase is $\tau_{r}$, and the reporting delay of each $\mathrm{SU}_{i}$ is $\tau_{r i} \cdot \tau_{r i_{s}}$ is the reporting delay of $\mathrm{SU}_{i}$ if $\mathrm{SU}_{i}$ reports local observation statistics, and $\tau_{r i h}$ is the reporting delay of $\mathrm{SU}_{i}$ if $\mathrm{SU}_{i}$ reports local decision. 


\section{Performance Analysis}

3.1. Energy Detection. Local spectrum sensing problem using energy detection can be formulated as the following binary hypothesis test:

$$
\begin{gathered}
H_{1}: y_{i}(n)=h_{i}(n) s_{i}(n)+u_{i}(n), \\
H_{0}: y_{i}(n)=u_{i}(n),
\end{gathered}
$$

where $H_{1}$ and $H_{0}$ denote that the PU on the channel is absent and present, respectively. $y_{i}(n)$ is the received signal at the $n$th sample in $\mathrm{SU}_{i}$. The noise $u_{i}(n)$ is a Gaussian, independent, and identically distributed random process with mean zero and variance $\sigma_{u}^{2}$. The primary signal $s_{i}(n)$ is a random process with mean zero and variance $\sigma_{s}^{2}$. We assume that the primary signal $s_{i}(n)$ and the noise $u_{i}(n)$ are both circularly symmetric complex Gaussian (CSCG) signal. $h_{i}(n)$ denotes the channel gain of the listening channel between the PU and $\mathrm{SU}_{i}$. Similar with the analysis in [7], We assume that $h_{i}(n)=h_{i}$. We denote $\gamma_{i}=\left|h_{i}\right|^{2} \sigma_{s}^{2} / \sigma_{u}^{2}$ as the signal-to-noise ratio (SNR) at the $\mathrm{SU}_{i}$ when PU exists and assume that the size of the CRN is small compared with its distance from the primary system; all SUs experience almost identical path loss with same average SNR on the listening channel, therefore $\gamma_{1}=\gamma_{2}=\cdots=\gamma_{i}=\cdots=$ $\gamma_{M}=\gamma=\bar{\gamma}$. According to the energy detection, we use the test statistic given by

$$
Y(i)=\frac{1}{N} \sum_{n=1}^{N}\left|y_{i}(n)\right|^{2}
$$

where $N$ denotes the number of samples. Using central limit theorem (CLT), for a large $N$, the probability density function (PDF) of $Y(i)$ under hypothesis $H_{0}$ can be approximated by a Gaussian distribution with mean $u_{0}=\sigma_{u}^{2}$ and variance $\sigma_{0}^{2}=(1 / N) \sigma_{u}^{4}$. The PDF of $Y(i)$ under hypothesis $H_{1}$ can be approximated by a Gaussian distribution with mean $u_{1}=$ $(\gamma+1) \sigma_{u}^{2}$ and variance $\sigma_{1}^{2}=(1 / N)(\gamma+1)^{2} \sigma_{u}^{4}$. When the local detection threshold is given by $\lambda_{l}$, the probability of local false alarm is given by

$$
P_{f_{\perp}}=\operatorname{Pr}\left[Y(i)>\lambda_{l} \mid H_{0}\right]=Q\left(\frac{\lambda_{l}-\sigma_{u}^{2}}{\sigma_{u}^{2}} \sqrt{N}\right) .
$$

The probability of local detection is given by

$$
P_{d l}=\operatorname{Pr}\left[Y(i)>\lambda_{l} \mid H_{1}\right]=Q\left(\frac{\lambda_{l}-(\gamma+1) \sigma_{u}^{2}}{(\gamma+1) \sigma_{u}^{2}} \sqrt{N}\right),
$$

where

$$
Q(x)=\frac{1}{\sqrt{2 \pi}} \int_{x}^{\infty} \exp \left(-\frac{t^{2}}{2}\right) d t .
$$

3.2. Hybrid Combination. In the hybrid combination system model, $\mathrm{SU}_{i}(i=K+1, K+2, \ldots, M)$ reports its local decision to FC. We define $\overline{P_{d}^{f c}}$ as the target probability of system detection with which the PUs are regarded as being sufficiently protected. When all the SUs report local decision to FC, according to the OR-rule in hard combination (To focus on the overhead-throughput tradeoff problem, we use ORrule for analysis simplification. The task of investigating other combination rules, e.g., the majority rule, is important but challenging, which would be studied in our future work.), the probability of system detection $\overline{P_{d}^{f c}}$ is given by

$$
\overline{P_{d}^{f c}}=1-\left(1-P_{d \downarrow}\right)^{M} \text {. }
$$

Then the probability of local detection $P_{d_{l}}$ is given by

$$
P_{d \perp}=1-\left(1-\overline{P_{d}^{f c}}\right)^{1 / M} .
$$

By substituting (7) into (4), the local detection threshold $\lambda_{l}$ is given by

$$
\lambda_{l}=(\gamma+1) \sigma_{u}^{2}\left(\frac{Q^{-1}\left(1-\left(1-\overline{P_{d}^{f c}}\right)^{1 / M}\right)}{\sqrt{N}+1}\right) .
$$

For $\mathrm{SU}_{i}(i=1,2, \ldots, K)$, following the analysis of soft cooperative energy detection in $[8,13]$, we use the test statistic given by

$$
T_{S-K}=\frac{1}{K N} \sum_{i=1}^{K} \sum_{n=1}^{N}\left|y_{i}(n)\right|^{2} .
$$

For large $K N$, according to CLT, the PDF of $T_{S-K}$ under hypothesis $H_{0}$ can be approximated as Gaussian with mean $\sigma_{u}^{2}$ and variance $(1 / K N) \sigma_{u}^{4}$. The PDF of $T_{S-K}$ under hypothesis $H_{1}$ can be approximated by a Gaussian distribution with mean $(\gamma+1) \sigma_{u}^{2}$ and variance $(1 / K N)(\gamma+1)^{2} \sigma_{u}^{4}$. The probability of detection with $K$ nodes soft combination $P_{d-K}$ is given by

$$
P_{d \_K}=\operatorname{Pr}\left[Y(i)>\lambda_{K} \mid H_{1}\right]=Q\left(\frac{\lambda_{K}-(\gamma+1) \sigma_{u}^{2}}{(\gamma+1) \sigma_{u}^{2}} \sqrt{K N}\right),
$$

where $\lambda_{K}$ is the soft combination detection threshold. According to the OR-rule, the relationship between $\overline{P_{d}^{f c}}$ and $P_{d \_K}$ is given by

$$
\overline{P_{d}^{f c}}=1-\left(1-P_{d \downarrow}\right)^{M-K}\left(1-P_{d \_K}\right) .
$$

Then we can get $P_{d_{-} K}$ by solving (11)

$$
P_{d_{-} K}=1-\frac{\left(1-\overline{P_{d}^{f c}}\right)}{\left(1-P_{d \downarrow}\right)^{M-K}} .
$$

According to (10), $\lambda_{K}$ is given by

$$
\lambda_{K}=(\gamma+1) \sigma_{u}^{2}\left(\frac{Q^{-1}\left(P_{d_{-} K}\right)}{\sqrt{K N}}+1\right) .
$$


By (3), the probability of false alarm of $K$ soft combination is given by

$$
P_{f-K}=\operatorname{Pr}\left[Y(i)>\lambda_{K} \mid H_{0}\right]=Q\left(\frac{\lambda_{K}-\sigma_{u}^{2}}{\sigma_{u}^{2}} \sqrt{K N}\right) .
$$

According to OR-rule and (14), the probability of system false alarm $P_{f}^{f_{c}}$ when $K$ SUs report local observation statistics is given by

$$
\begin{aligned}
& P_{f}^{f c} \\
& =1-\left(1-P_{f_{-} l}\right)^{M-K}\left(1-P_{f_{-} K}\right) \\
& =1-\left(1-P_{f_{-} l}\right)^{M-K} \\
& \quad \times(1-Q \\
& \left.\quad \times\left(\frac{(\gamma+1) \sigma_{u}^{2}\left(Q^{-1}\left(P_{d_{-} K}\right) / \sqrt{K N}+1\right)-\sigma_{u}^{2}}{\sigma_{u}^{2}} \sqrt{K N}\right)\right),
\end{aligned}
$$

where $P_{d_{-} K}, P_{f \perp}$, and $P_{d \perp}$ are related to $\overline{P_{d}^{f c}}$, given by (12), (3), and (4), respectively.

3.3. The Throughput. We assume that the PU is either absent or present during each frame duration (Figure 2). The achievable throughput of the secondary network is given by

$$
C=\frac{T-\tau_{s}-\tau_{r}}{T}\left(1-P_{f}^{f c}\right) C_{0} \operatorname{Pr}\left(H_{0}\right),
$$

where $\operatorname{Pr}\left(H_{0}\right)$ is the probability for which the PU is inactive. $C_{0}$ is the throughput of the secondary network when it operates in the absence of PU.

We assume that the local observation statistics are quantized with $D$ bits, which would be a sufficient number of bits to neglect the quantization noise [11]. If $\mathrm{SU}_{i}$ reports its local observation statistics to $\mathrm{FC}$, the reporting delay would be $\tau_{r i_{s} s}=D / R_{i}$. If $\mathrm{SU}_{i}$ reports its local decision to $\mathrm{FC}$, the reporting delay would be $\tau_{r i \_}=1 / R_{i}$. When $\mathrm{SU}_{i}(i=$ $1,2, \ldots, K)$ reports its local observation statistics to FC and $\mathrm{SU}_{i}(i=K+1, K+2, \ldots, M)$ reports its local decision to FC, according to the frame structure in Figure 2, the total reporting delay is

$$
\tau_{r}=\sum_{i=1}^{K} \tau_{r i_{-} s}+\sum_{i=K+1}^{M} \tau_{r i_{-} h}=\sum_{i=1}^{K} \frac{D}{R_{i}}+\sum_{i=K+1}^{M} \frac{1}{R_{i}} .
$$

The throughput is given by

$$
\begin{aligned}
C= & \frac{T-\tau_{s}-\sum_{i=1}^{K}\left(D / R_{i}\right)+\sum_{i=K+1}^{M}\left(1 / R_{i}\right)}{T} \\
& \times\left(1-P_{f}^{f c}\right) C_{0} \operatorname{Pr}\left(H_{0}\right) .
\end{aligned}
$$

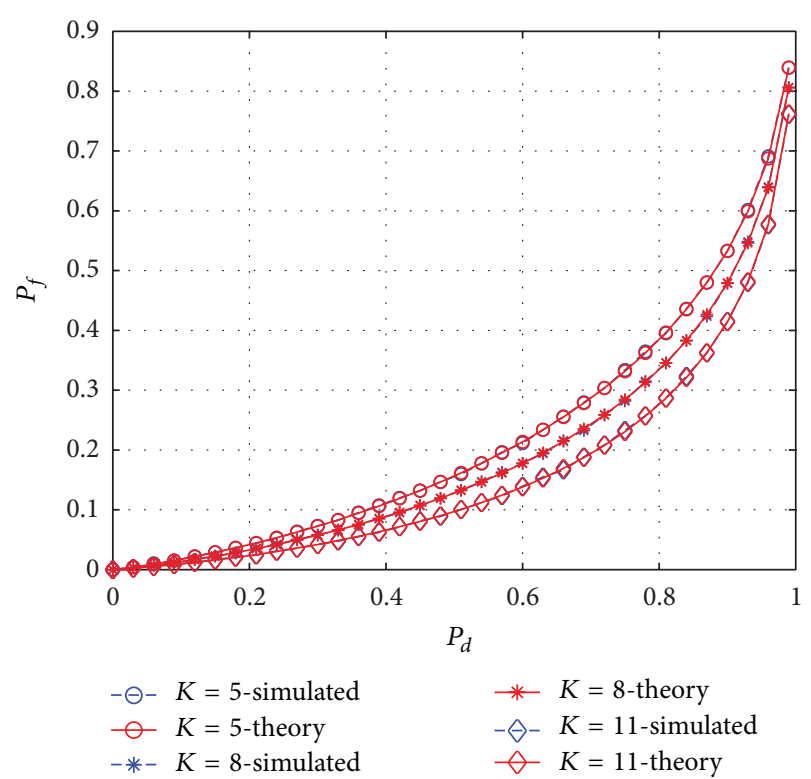

FIGURE 3: The probability of detection versus probability of false alarm with different $K$ when $\mathrm{SNR}=-22 \mathrm{~dB}$.

Intuitively, there is a tradeoff between $P_{f}^{f c}$ and $\tau_{r}$. When $K$ increases, the $P_{f}^{f c}$ decreases (Figure 3), which means the secondary network can access the channel with a higher chance, but the reporting delay also increases at the same time. The relationship between $K$ and the throughput $C$ is obtained by substituting (15) into (18)

$$
\begin{aligned}
C= & \frac{T-\tau_{s}-\sum_{i=1}^{K}\left(D / R_{i}\right)+\sum_{i=K+1}^{M}\left(1 / R_{i}\right)}{T}\left(1-P_{f\lrcorner}\right)^{M-K} \\
& \times C_{0} \operatorname{Pr}\left(H_{0}\right) \\
& \times(1-Q \\
& \left.\quad \times\left(\frac{(\gamma+1) \sigma_{u}^{2}\left(Q^{-1}\left(P_{d_{-} K}\right) / \sqrt{K N}+1\right)-\sigma_{u}^{2}}{\sigma_{u}^{2}} \sqrt{K N}\right)\right),
\end{aligned}
$$

where $P_{d_{-} K}, P_{f \perp}$, and $P_{d \perp}$ are related to $\overline{P_{d}^{f c}}$, given by (12), (3), and (4), respectively, and $\overline{P_{d}^{f c}}$ is determined by the protection demand of PU system.

\section{Numerical Results and Discussions}

4.1. Numerical Results. The system is set up as follows: the number of SUs in the CRN is set to be $M=18$, and the fixed frame duration $T=20 \mathrm{~ms}$. The sampling frequency is $6 \mathrm{MHz}$ and the $\tau_{s}$ is $1 \mathrm{~ms}$. We set quantization bits of observation statistics $D=8$ in order to ensure that the quantization can well preserve the local observation statistics. $D$ is bigger than that used in [11], because there is still some quantization noise 


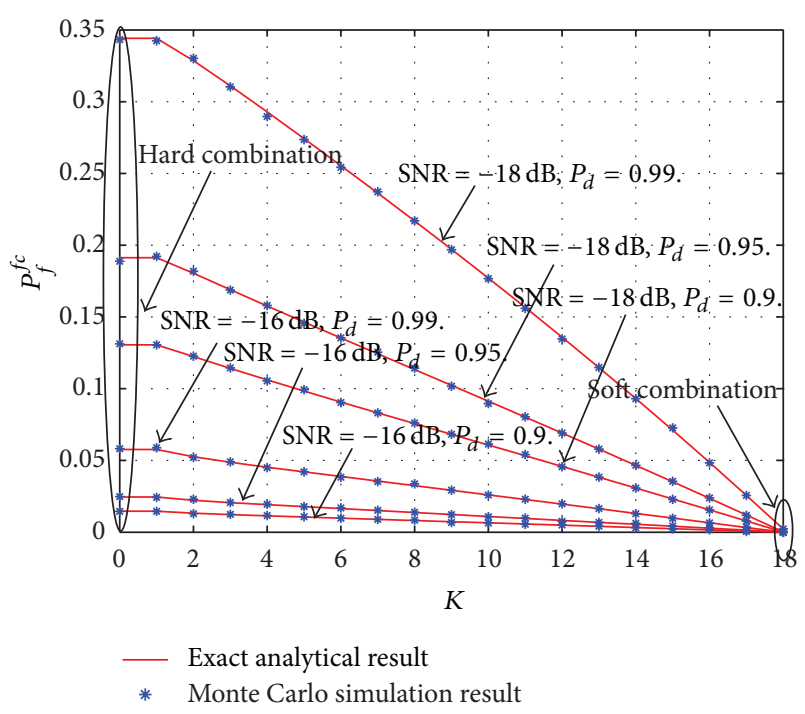

FIGURE 4: The false alarm probability versus the number of SUs $K$ which report local observation statistics in different settings.

in [11] which decreases with $D$; we do not focus on the analysis of quantization, so we use the sufficient quantization bits to ensure (11) is achievable.

Figure 3 shows the detection performance of the proposed hybrid detector (the probability of detection versus probability of false alarm). The result verifies the detection performance of the proposed hybrid detector. The hybrid detector works as the single detector at any $K$ values: the probability of detection increases as the probability of false alarm grows.

In addition, the performance grows better when $K$ grows. This result is reasonable because the more SUs report their local observation statistics, the more information FC gets.

Figure 4 shows the detection performance of the hybrid combination scheme. The detection performance grows better when the SNR increases, and the probability of false alarm increases when the probability of system detection $\overline{P_{d}^{f c}}$ increases (we choose the probability of system detection $\overline{P_{d}^{f c}}$ $0.9,0.95,0.99$ as typical values). When all the SUs report local observation statistics ( $K=M$, soft combination), the detection performance is best. When all the SUs report local observation statistics ( $K=0$, hard combination), the detection performance is worst. The result means that the hybrid combination method is reasonable $\left(P_{f}^{f c}\right.$ decreases when $K$ increases). In other words, in the hybrid combination scheme, when the number of the SUs reporting their local observation statistics to FC increases, the accuracy of sensing result will be enhanced.

The results in Figures 3 and 4 verify the proposed hybrid detector's reasonability, and the advantage of the proposed scheme over present scheme is the throughput when the reporting overhead is considered (Figures 5 and 6).

Figure 5 shows the throughput when using the reporting rate vector (RRV) values shown in Table 1 , which is
TABLE 1

\begin{tabular}{lc}
\hline No. & Reporting rate vector $(\mathrm{RRV})(\mathrm{kbit} / \mathrm{s})$ \\
\hline 1 & {$[30,30,30,30,30,30,20,20,20,20,20,20,15,15,15,15$,} \\
$15,15]$ \\
2 & {$[100,100,100,100,100,100,100,100,100,100,10,10,10$,} \\
& $10,10,10,10,10]$ \\
3 & {$[20,20,20,20,20,20,20,20,20,20,20,20,20,20,20$,} \\
& $20,20,20]$ \\
\hline
\end{tabular}

TABLE 2

\begin{tabular}{|c|c|}
\hline No. & Reporting rate vector (RRV) (kbit/s) \\
\hline 1 & $\begin{array}{c}{[20,20,20,20,20,20,20,20,20,20,20,20,20,20,20} \\
20,20,20] ; \sigma=0\end{array}$ \\
\hline 2 & $\begin{array}{c}{[30,30,30,30,30,30,20,20,20,20,20,20,16,16,16,16} \\
16,16] ; \sigma=6.0585\end{array}$ \\
\hline 3 & $\begin{array}{c}{[40,40,40,40,40,40,40,40,40,12,12,12,12,12,12,12} \\
12,12] ; \sigma=14.4059\end{array}$ \\
\hline 4 & $\begin{array}{c}{[50,50,50,50,40,40,40,40,40,13,13,13,13,13,13,13} \\
13,13] ; \sigma=18.9185\end{array}$ \\
\hline 5 & $\begin{array}{c}{[70,70,70,70,70,70,70,70,70,12,12,12,12,12,12,12} \\
12,12] ; \sigma=29.6560\end{array}$ \\
\hline 6 & $\begin{array}{c}{[90,90,90,90,90,90,90,90,90,10,10,10,10,10,10,10} \\
10,10] ; \sigma=40.9048\end{array}$ \\
\hline 7 & $\begin{array}{c}{[100,100,100,100,100,100,100,100,100,9,9,9,9,9,9} \\
9,9,9] ; \sigma=46.5292\end{array}$ \\
\hline
\end{tabular}

similar with the simulation setting in [11]. The throughput first increases as $K$ grows, resulting from the decrease of the probability of system false alarm. Then the throughput decreases because the overhead increases fast when SUs with bad reporting channel report their observation statistics to FC rather than local decisions. We can find the best $K\left(K^{*}\right)$ to obtain the best performance in the proposed hybrid combination scheme, which would outperform both hard combination and soft combination.

Figure 6 shows the performance comparison between the proposed hybrid combination scheme and the exiting combination scheme, that is, hard combination scheme, adopted by [12] and soft combination scheme, adopted by [5], also in $[7,13]$. The reporting rate vector (RRV) values are shown in Table 2; we denote $\sigma$ as the standard deviation of RRV. The value of $\sigma$ shows the diversity of reporting channel quality.

With different RRVs, the result of comparison between soft and hard combination scheme varies, which means the diversity of reporting channel quality has great influence on the choice of combination scheme.

However, the result shows that the proposed hybrid combination scheme outperforms both hard combination scheme [12] and soft combination scheme [5] in different environments, especially when the diversity of channel quality is obvious ( $\sigma$ increases). There is also throughput improvement when the data rates are the same ( $\sigma$ is zero). 


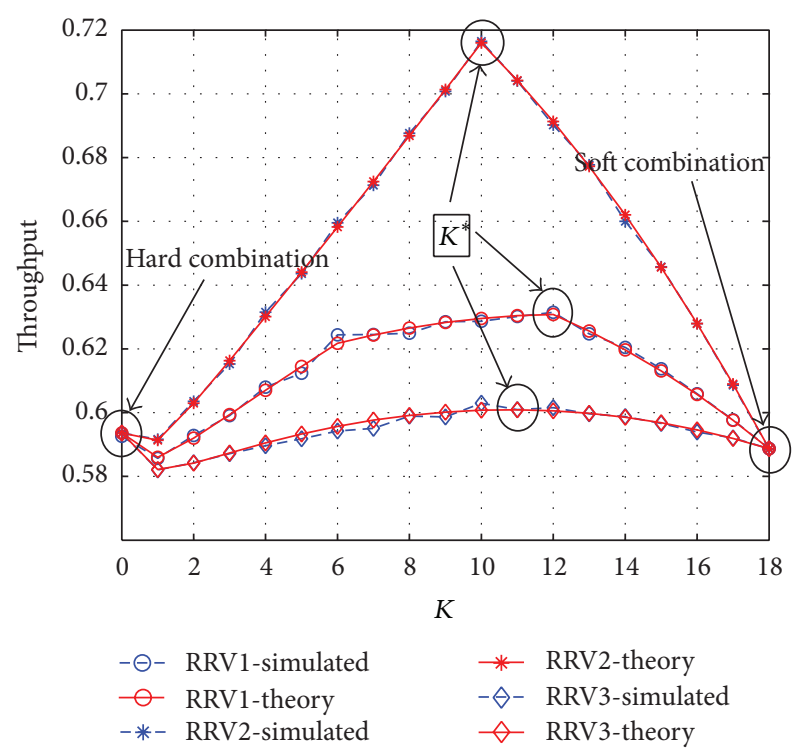

FIGURE 5: The throughput versus the number of SUs $K$ which report local observation statistics in different settings, $\overline{\left(P_{d}^{f c}\right.}$ is $0.99, \gamma$ is $-18 \mathrm{~dB})$.

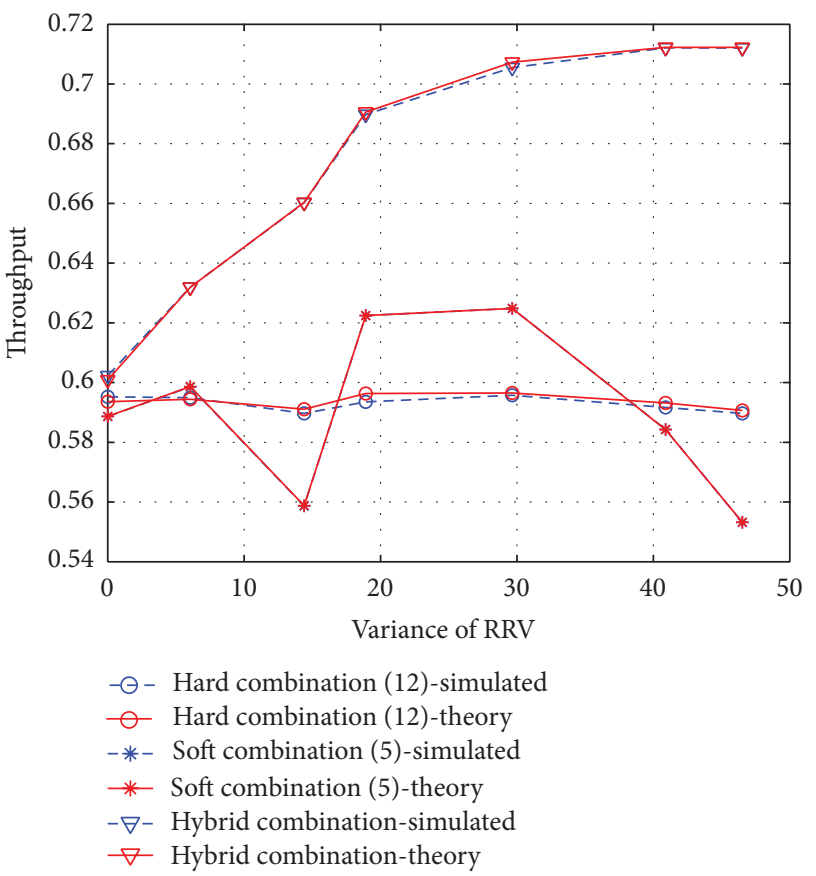

FIgURE 6: Performance comparison between the proposed hybrid combination scheme and the exiting scheme with different reporting rate vectors.

The explanation for the better performance is that we consider the diversity of reporting channel which has not been taken into consideration in present works.

4.2. Implementation and Complexity Analysis. According to $R$ obtained by the negotiation when the cooperative relationship between SUs and FC is setting up, FC can find the $K^{*}$ according to (19). The computing complexity is low because FC only needs to compute $M$ (number of SUs) values of (19), and $M$ would not be large in the practical cooperative system. Then the FC obtains a reporting model vector (RMV) composed of $M$ bits; the $i$ th bit of the RMV $\left(\mathrm{RMV}_{i}\right)$ denotes the reporting model of $\mathrm{SU}_{i} \cdot \mathrm{RMV}_{i}=0$ means $\mathrm{SU}_{i}$ reports its local decision, and $\mathrm{RMV}_{i}=1$ means $\mathrm{SU}_{i}$ reports its observation statistics. Just like the cooperative system in [12], at the beginning of each frame, the FC would broadcast a beacon message during beacon period (BP) which contains the start time and length of the quiet period (QP) for channel sensing, and the RMV would be attached to the beacon message. In this way, the overhead of the hybrid combination is only $M$ bits attached to the broadcasted beacon message. In all, the computing complexity and the additional overhead of the hybrid combination are very slight.

\section{Conclusions}

In this paper, we investigated overhead-throughput tradeoff over different reporting channel quality in CRN that consists of a number of the SUs employing energy detectors and a single decision fusion center. We proposed a novel hybrid combination scheme with low overhead and complexity, which outperforms both hard combination and soft combination scheme, especially when the diversity of the reporting channel is obvious.

\section{Conflict of Interests}

The authors declare that they have no conflict of interests regarding the publication of this paper.

\section{Acknowledgments}

This work was supported by the National Natural Science Foundation of China (Grant nos. 61172062, 61301160), Jiangsu Province Natural Science Foundation (Grant no. BK2011116), and in part by the National Basic Research Program of China (Grant no. 2009CB320400).

\section{References}

[1] J. Mitola III and G. Q. Maguire Jr., "Cognitive radio: making software radios more personal," IEEE Personal Communications, vol. 6, no. 4, pp. 13-18, 1999.

[2] S. Haykin, "Cognitive radio: brain-empowered wireless communications," IEEE Journal on Selected Areas in Communications, vol. 23, no. 2, pp. 201-220, 2005.

[3] A. Ghasemi and E. S. Sousa, "Collaborative spectrum sensing for opportunistic access in fading environments," in Proceedings of the 1st IEEE International Symposium on New Frontiers in Dynamic Spectrum Access Networks (DySPAN '05), pp. 131-136, November 2005.

[4] Z. Quan, S. Cui, and A. H. Sayed, "Optimal linear cooperation for spectrum sensing in cognitive radio networks," IEEE Journal on Selected Topics in Signal Processing, vol. 2, no. 1, pp. 28-40, 2008. 
[5] H. Yu, W. Tang, and S. Li, "Optimization of cooperative spectrum sensing with sensing user selection in cognitive radio networks," EURASIP Journal on Wireless Communications and Networking, vol. 2011, article 208, 8 pages, 2011.

[6] B. A. Bastami and E. Saberinia, "A practical multibit data combining strategy for cooperative spectrum sensing," IEEE Transactions on Vehicular Technology, vol. 62, no. 1, pp. 384-389, 2013.

[7] W. Zhang, R. K. Mallik, and K. Ben Letaief, "Optimization of cooperative spectrum sensing with energy detection in cognitive radio networks," IEEE Transactions on Wireless Communications, vol. 8, no. 12, pp. 5761-5766, 2009.

[8] J. Shen, S. Liu, R. Zhang, and Y. Liu, "Soft versus hard cooperative energy detection under low SNR," in Proceedings of the $3 r d$ International Conference on Communications and Networking in China (ChinaCom '08), pp. 128-131, August 2008.

[9] W. Xia, W. Yuan, W. Cheng, W. Liu, S. Wang, and J. Xu, "Optimization of cooperative spectrum sensing in ad-hoc cognitive radio networks," in Proceedings of the 53rd IEEE Global Communications Conference (GLOBECOM '10), pp. 1-5, December 2010.

[10] L.-C. Wang, Y.-C. Lu, C.-W. Wang, and D. S. L. Wei, "Latency analysis for dynamic spectrum access in cognitive radio: dedicated or embedded control channel?" in Proceedings of the 18th Annual IEEE International Symposium on Personal, Indoor and Mobile Radio Communications (PIMRC '07), pp. 1-5, September 2007.

[11] Y. Tani and T. Saba, "Quantization scheme for energy detector of soft decision cooperative spectrum sensing in cognitive radio," in Proceedings of the IEEE Global Telecommunications Conference, pp. 69-73, December 2010.

[12] W. S. Jeon, D. H. Lee, and D. G. Jeong, "Collaborative sensing management for cognitive radio networks with reporting overhead," IEEE Transactions on Wireless Communications, vol. 12, no. 2, pp. 595-605, 2013.

[13] Y.-C. Liang, Y. Zeng, E. C. Y. Peh, and A. T. Hoang, "Sensingthroughput tradeoff for cognitive radio networks," IEEE Transactions on Wireless Communications, vol. 7, no. 4, pp. 1326-1337, 2008. 


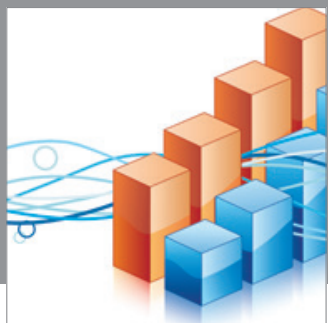

Advances in

Operations Research

mansans

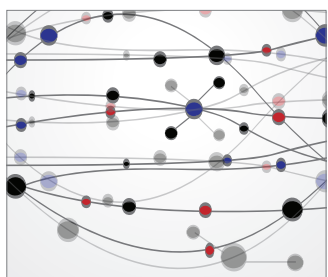

The Scientific World Journal
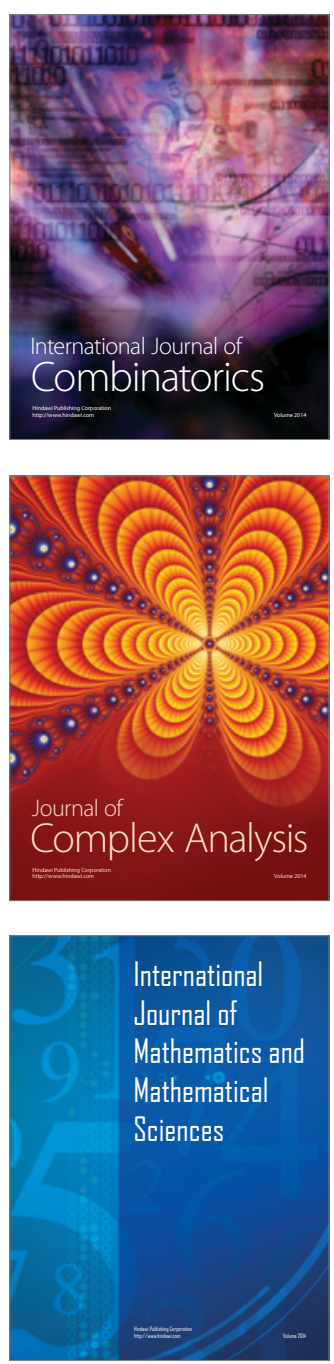
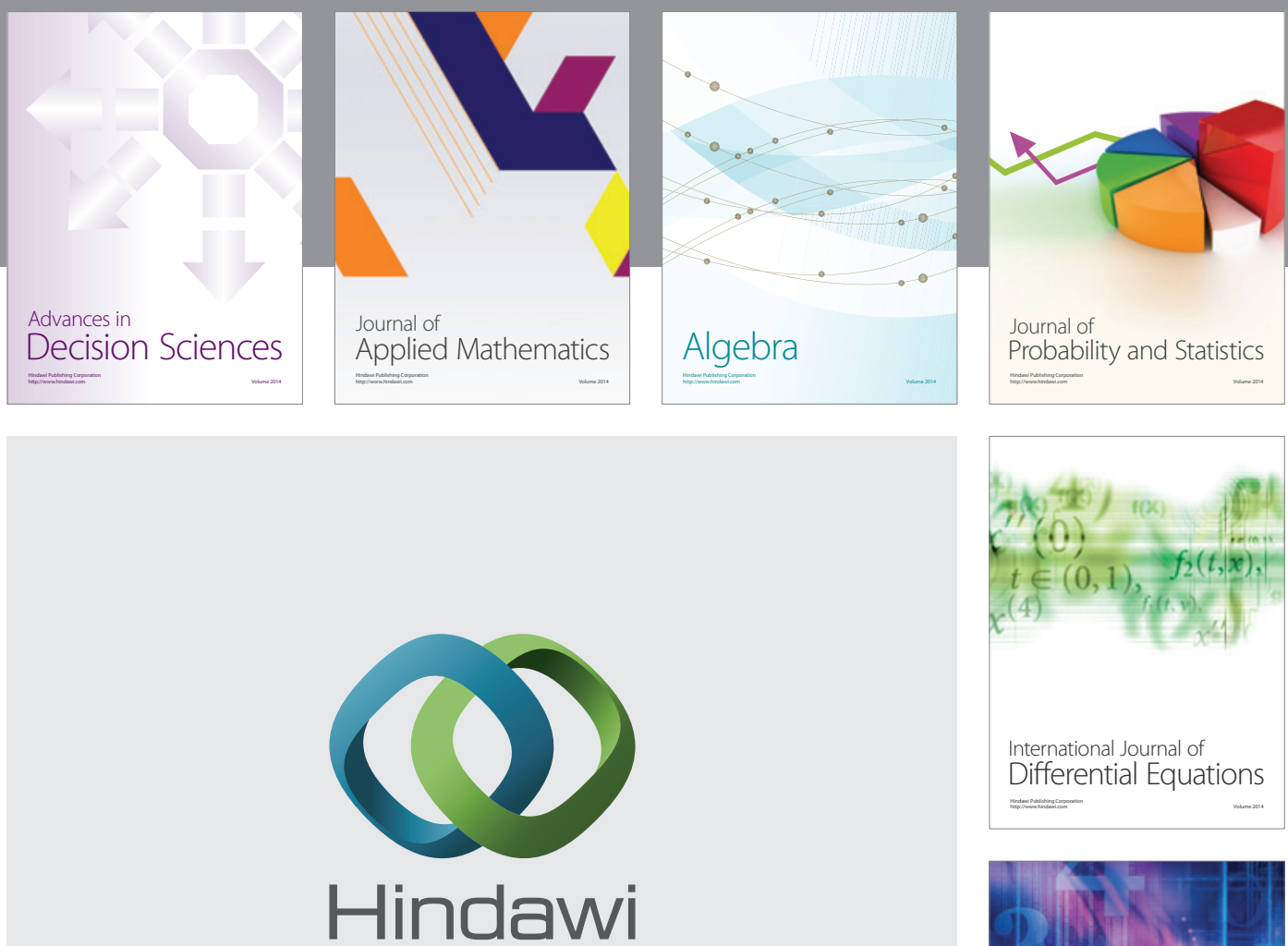

Submit your manuscripts at http://www.hindawi.com
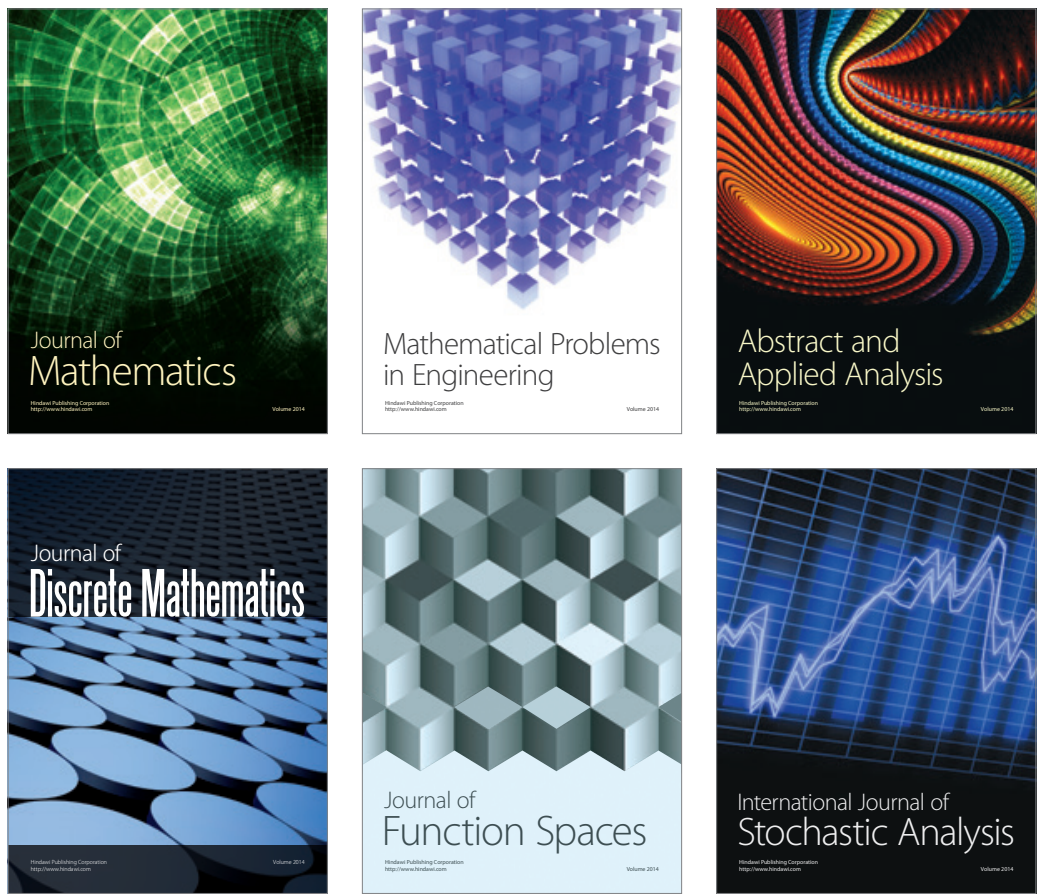

Journal of

Function Spaces

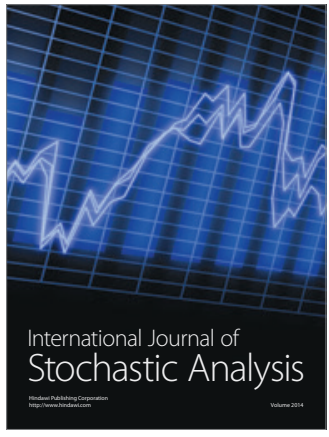

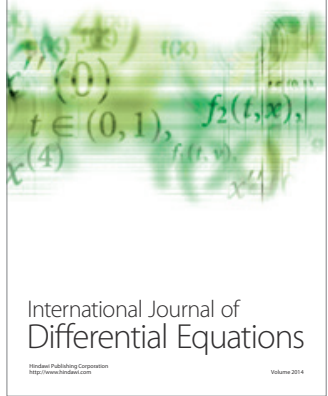
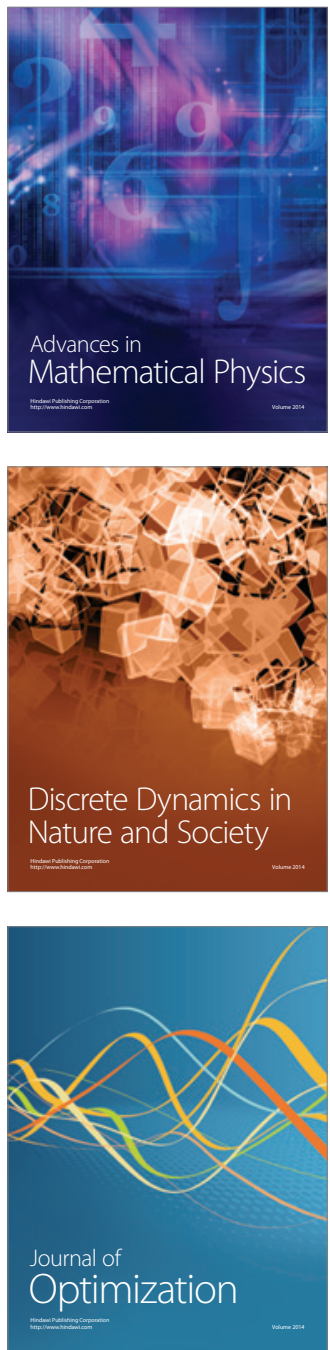